\title{
The MiRNA Journey from Theory to Practice as a CNS Biomarker
}

\author{
Nicoleta Stoicea ${ }^{1 *}$, Amy $\mathrm{Du}^{2}$, D. Christie Lakis ${ }^{2}$, Courtney Tipton ${ }^{2}$, \\ Carlos E. Arias-Morales ${ }^{1}$ and Sergio D. Bergese ${ }^{1,3}$
}

${ }^{1}$ Department of Anesthesiology, The Ohio State University Wexner Medical Center, Columbus, OH, USA, ${ }^{2}$ College of Medicine, The Ohio State University, Columbus, OH, USA, ${ }^{3}$ Department of Neurological Surgery, The Ohio State University Wexner Medical Center, Columbus, OH, USA

\section{OPEN ACCESS}

Edited by:

Subbaya Subramanian, University of Minnesota, USA

Reviewed by:

Venugopal Thayanithy, University of Minnesota, USA Reena V. Kartha,

University of Minnesota, USA

*Correspondence:

Nicoleta Stoicea

nicoleta.stoicea@osumc.edu

Specialty section:

This article was submitted to

RNA

a section of the journal

Frontiers in Genetics

Received: 23 November 2015

Accepted: 24 January 2016

Published: 09 February 2016

Citation:

Stoicea N, Du A, Lakis DC, Tipton C, Arias-Morales CE and Bergese SD (2016) The MiRNA Journey from

Theory to Practice as a CNS

Biomarker. Front. Genet. 7:11

doi: 10.3389/fgene.2016.00011
MicroRNAs (miRNAs), small nucleotide sequences that control gene transcription, have the potential to serve an expanded function as indicators in the diagnosis and progression of neurological disorders. Studies involving debilitating neurological diseases such as, Alzheimer's disease, multiple sclerosis, traumatic brain injuries, Parkinson's disease and CNS tumors, already provide validation for their clinical diagnostic use. These small nucleotide sequences have several features, making them favorable candidates as biomarkers, including function in multiple tissues, stability in bodily fluids, a role in pathogenesis, and the ability to be detected early in the disease course. Cerebrospinal fluid, with its cell-free environment, collection process that minimizes tissue damage, and direct contact with the brain and spinal cord, is a promising source of miRNA in the diagnosis of many neurological disorders. Despite the advantages of miRNA analysis, current analytic technology is not yet affordable as a clinically viable diagnostic tool and requires standardization. The goal of this review is to explore the prospective use of CSF miRNA as a reliable and affordable biomarker for different neurological disorders.

Keywords: MicroRNA, biomarker, cerebrospinal fluid (CSF), central nervous system (CNS), neurodegenerative disease

\section{INTRODUCTION}

MicroRNAs (miRNA) are small, noncoding RNA fragments approximately 22 nucleotides long involved in post-transcriptional regulation of gene expression (Guan et al., 2010; Gallego et al., 2012; Cheng et al., 2013). After processing by endonucleases (Cheng et al., 2013; Freischmidt et al., 2013), the resultant single-stranded miRNAs combine with other macromolecules to form RNAinduced silencing complexes (RISC). These RISCs target complementary messenger RNA (mRNA) strands for degradation, altering cellular function (Cheng et al., 2013; Freischmidt et al., 2013).

MiRNAs are thought to have widespread action as more than 1500 identified miRNAs have been shown to affect the expression of 30-60\% or more of all genes (Siegel et al., 2012; Ksiazek-Winiarek et al., 2013; Sheinerman and Umansky, 2013; Cloutier et al., 2015). MiRNA plays a role in virtually all cellular functions, such as cell proliferation, differentiation, and importantly apoptosis (Guan et al., 2010; Siegel et al., 2012; Ksiazek-Winiarek et al., 2013). Additionally, miRNAs are widely present in all body tissues and fluids such as plasma, serum, urine, saliva, milk and CSF (Siegel et al., 2012; Galimberti et al., 2014).

Various miRNAs subtypes are differently expressed among the organs of the body and within the organs themselves. For example, a patient with Alzheimer's disease (AD) will express a different miRNA profile in the medial frontal gyrus, hippocampus, and cerebellum (Cogswell et al., 2008). 
Due to its pervasiveness, stability in bodily fluids, and dysregulation in early disease course, miRNA could be considered a reliable biomarker.

In our analysis, we explored the recent history of serum and CSF protein biomarkers, discussed the advantages of CSF miRNA, and examined the utility of CSF miRNA in CNS disease management.

\section{METHODS}

In assessing the reliability of miRNA as a biomarker, we conducted an extended review of literature on CSF miRNA in CNS disorders, published within the last 5 years. Choice landmark studies were also included.

\section{Serum and CSF Protein Biomarkers}

Serum and CSF protein biomarkers are able to provide a fast and accurate diagnosis of various diseases. For example, CSF IL-8 and serum TNF $\alpha$, are considered sensitive markers of neuroinflammation (Maxeiner et al., 2014). Furthermore, another well-studied CSF biomarker, IL-6, along with IL- 8 have been found to be elevated in patients with depression and delirium (van Munster et al., 2008; van den Boogaard et al., 2011; Liu et al., 2013; Kern et al., 2014).

CSF and serum biomarkers also have shown predictive value in evaluating neurologic disease progression. Czeiter et al. demonstrated that, serum and CSF GFAP_24S levels facilitated traumatic brain injury prognosis alongside the IMPACT calculator (Czeiter et al., 2012). Several studies have also investigated $\mathrm{AD}$ biomarker prognostic potential, including the ability to track cognitive decline post-surgery and progression into AD (Ackermann et al., 2014). Two such CSF biomarkers, neurogranin and tryptic peptide SNAP25, should be able to track AD progression. Furthermore, neurogranin specifically identifies MCI patients at risk for developing AD (Brinkmalm et al., 2014; Kvartsberg et al., 2014).

CSF protein biomarkers, $\alpha 2 \mathrm{HS}$ glycoprotein, $\alpha 1$ antichymotrypsin, and albeta glycoprotein, may predict the benefit of ventriculoperitoneal shunt for patients with normal pressure hydrocephalus (NPH) (Scollato et al., 2010). $\mathrm{NPH}$ patients with improved shunt response also have been linked to significantly lower CSF levels of sAPP $\alpha$ and sAPP $\beta$ (Pyykö et al., 2014).

Although these protein biomarkers have promising diagnostic, prognostic, and therapeutic benefits, recent literature explores their limitations. Interestingly, only $77.2 \%$ of $2357 \mathrm{AD}$ patients expressed disease related CSF $\mathrm{A} \beta$ and either T-tau or P-tau protein values (Hoglund et al., 2015; Rosén et al., 2015). MiRNA appears to predict and diagnose $\mathrm{AD}$ with greater sensitivity, even during mild cognitive impairment. For example, Alexandrov et al. noticed miR-9, miR-125b, miR-146b, and miR-155 upregulation in the diseased state when no significant correlation was established between $\mathrm{A} \beta$ and $\mathrm{AD}$ (Alexandrov et al., 2012).

\section{Advantages of Using miRNA as a Biomarker}

Because of its widespread action and presence, miRNA profile changes enable the identification of many pathological processes, including malignancies and cardiovascular and neurological diseases (Calin et al., 2002; Bonauer et al., 2010; Siegel et al., 2012).

MiRNAs' stability is a promising biomarker quality. Interestingly, Siegel demonstrated a small percent of circulating miRNAs are protected from degradation due to their inclusion in small vesicles known as exomes; furthermore, peripheral miRNAs included in RISC complexes avoid degradation by RNAses (Siegel et al., 2012). MiRNA is unique in playing a central role in gene regulation. Schramedei et al. found miR21 to be a key oncogenic regulator, downregulating tumor suppressors ANP32A and SMARCA4 (Schramedei et al., 2011). Thus, miR-21 could be a more reliable indicator of disease progression than other tangential biomarkers in the progression of cancers, like CNS tumors. CSF elevation of let-7b, a miRNA precursor, is correlated with $\mathrm{AD}$. When administered in vitro, let-7b was shown to proportionally decrease neuronal survival while sparing cell lines containing mutated TLR7 or downstream proteins. Lehmann et al. therefore concluded that let- $7 \mathrm{~b}$ causes neurodegeneration in $\mathrm{AD}$ through activation of Toll-like Receptor 7 (Lehmann et al., 2012). Ghidoni et al. linked a decreased miR-107 in early AD to BACE1, a pathogenic compound in AD (Ghidoni et al., 2011). Unfortunately AD clinical manifestations and post-mortem diagnosis often overlap with other forms of dementia such as frontotemporal or Lewy body dementia (Humpel, 2011). Moreover, observable symptoms manifest late in the clinical course impairing early diagnosis and prevention. Other potential biomarkers often appear in much later stages of the disease when neuronal damage has already occurred (Kim et al., 2014). Similar to AD, amyotrophic lateral sclerosis (ALS) identification also relies heavily on clinical presentation, responsible for misdiagnosis in $8-44 \%$ of cases (Cloutier et al., 2015).

\section{CSF As a Source of miRNA}

In patients with neurological disorders, CSF is the ideal source of miRNA, its profile being identical to that of the brain tissue because of its close proximity. CSF sampling from lumbar puncture is often standard procedure for the diagnosis of MS and ALS (Polman et al., 2005; The ALS Association, 2015). Even though tissue biopsy may be more accurate than CSF collection, CSF sampling has the benefit of being much less invasive.

\section{CSF Processing}

Method of extraction of CSF usually varies. As a standard procedure, after CSF is collected, centrifugation $(500 \times \mathrm{g}, 10 \mathrm{~min}$, and room temperature) needs to be performed within $60 \mathrm{~min}$ after collection in order to remove cells and debris. Storage of centrifuged samples is usually at $-80^{\circ} \mathrm{C}$. Baraniskin et al. (2011) MicroRNA can be extracted using different kits on the market, and manufacturer instructions have to be followed carefully in order to yield the maximum RNA possible. Although $1 \mathrm{~mL}$ of CSF only yields $15-30$ ng of total RNA, Burgos et al. reported that is 
possible to obtain reproducible results from as little as $0.5 \mathrm{~mL}$ of human CSF (Burgos et al., 2013).

MiRNA can also be isolated from a multitude of blood sources, including serum, plasma, leukocytes, and whole blood samples, establishing its effectiveness as a biomarker (Cheng et al., 2013; De Felice et al., 2014). While both CSF and serum miRNA correlate well with many pathological processes, blood sources are often preferred over CSF sources because they are less invasive and accessible (De Felice et al., 2014). Within the context of neurological disorders, however, CSF typically yields more accurate results (Freischmidt et al., 2013; Cloutier et al., 2015). A meta-analysis examining data from over 20 original works on CNS malignancies found that CSF-based miRNA profile was more sensitive and specific than blood-derived miRNA. In this meta-analysis, the sensitivity for detecting CNS malignancies increased from $83 \%$ for blood-derived miRNA to $86 \%$ for CSF miRNA while the specificity increased from $81 \%$ in blood to $88 \%$ in CSF (Wei et al., 2015).

Blood volume is greater than CSF volume and thus, miRNA concentration is diluted when the nucleotide sequences cross from the CSF through the blood-brain barrier (BBB) and into the blood. This dilution process leads to less clarity in the diagnostic findings. Furthermore, while CSF contains miRNAs shed directly from the brain, blood contains miRNAs shed from all bodily tissues. For example, peripheral tissues excrete large amounts of miR-21 that can interfere with the diagnosis CNS tumors which also excrete miR-21 (Akers et al., 2013). In the case of miR21 excreting CNS tumors, CSF sampling is therefore preferred. While certain miRNA exist in both blood and CSF, they are often poorly correlated. Furthermore, other miRNA subtypes have been exclusively identified in CSF or blood. Burgos et al. also compared microRNA present in serum and CSF from five subjects, and the study revealed that profiles of microRNA are more similar from different subjects for either CSF or serum and are less similar to one another, even within the same subject. The reasoning of this finding is due to microRNAs in CSF are derived from neural cells, whereas serum microRNAs are collected from all tissues in the body. The authors concluded based on the results, that while there are a large number of microRNA unique to CSF and serum, most miRNAs are present in both of them (Burgos et al., 2013). Also, sequencing small RNAs from small volumes can provide a large amount of reproducible data.

Early studies seem to suggest that the BBB plays a minor role in dictating the passage of miRNA between CSF and blood. The BBB becomes more permeable as a result of the inflammatory component of many neurodegenerative diseases. This process is not well understood and it seems at baseline that the BBB could still have residual effects in preventing the mixing of CSF and blood (Blennow et al., 1990; Berzin et al., 2000; Begley and Brightman, 2003; Zipser et al., 2007; Cheng et al., 2013). Other sources of miRNA such as urine, milk, and saliva may be less invasive and cost-effective (Cheng et al., 2013; Sheinerman and Umansky, 2013). However, they may contain more contaminants. Urine, for example, tends to be very rich in cells and can contain microorganisms in addition to renal, hematologic, and other cellular debris, providing insufficient or unsatisfactory data (Cheng et al., 2013).

\section{DISCUSSION}

\section{CNS Degenerative Diseases}

Abnormal CSF miRNA profiles could diagnose various CNS disorders, such as AD. Cogswell et al. was one of the earliest to discover that CSF miRNA profiles could differentiate AD from control patients. CSF was isolated from 20 donors, including Braak Stage 5 (severe AD) and Braak Stage 1 (nondemented controls). MiR-27a, miR-125b, miR-146, and 57 others were significantly upregulated or downregulated in the Braak 5 population relative to the Braak 1 population. These dysregulated miRNAs were found to be involved in immune cell function, $\mathrm{T}$ cell signaling, and T cell activation (Cogswell et al., 2008).

Recent studies have validated the use of CSF miRNA in $\mathrm{AD}$ detection. Galimberti et al. studied $22 \mathrm{AD}$ patients and 18 Non-inflammatory neurological disease controls (NINDC). In a confirmatory study, they evaluated $15 \mathrm{AD}$ and 12 NINDC patients. They found significantly decreased levels of CSF miR-125b and miR-26b in AD patients compared to controls (Galimberti et al., 2014). Likewise, miR-802, which is located in the RUNX1 gene (greatly overexpressed in AD) has been shown to be correlated with early AD (van Harten et al., 2011).

Galimberti et al. reported a negative correlation of CSF miR$26 \mathrm{~b}$ with total and phosphorylated Tau (T-Tau and P-Tau) levels in AD patients (Galimberti et al., 2014). Sala et al. analyzed 8 miRNAs from 2 age-matched cohorts and found 1-fold lower CSF miR-27a-3p expression and 1-fold higher miR-216a-5p and miR210 expression in $\mathrm{AD}$ patients. Levels of these miRNA were also correlated with T-tau and P-tau values (Sala Frigerio et al., 2013). It is encouraging that many of these CSF miRNAs correlate with proteins that are virtually pathognomonic for AD such as Tau and $A \beta$. The dysregulation of miR-29b and miR-125b were further studied in the CSF of 18 AD patients and 20 healthy controls using quantitative PCR. CSF miR-29a levels were increased by a factor of 2.2 in $\mathrm{AD}$ patients when compared to controls (Müller et al., 2015). MiR-29a is thought to play a part in the formation of $\mathrm{BACE} 1$, a key enzyme involved in $\mathrm{A} \beta$ amyloid formation (Hébert et al., 2008). AD differentiation from healthy controls using miR29 a a biomarker had $89 \%$ sensitivity and $70 \%$ specificity. MiR-125b expression was slightly increased, with 78\% sensitivity and $60 \%$ specificity in differentiating $\mathrm{AD}$ patients from controls (Müller et al., 2015). MiR-125b is thought to be involved in the cell growth and differentiation pathway via Hedgehog signaling (Ferretti et al., 2008).

Given new evidence for its potential role in hypoxic injury and VEGF (an angiogenic growth factor) mediated disease, miR-210 expression was quantified in 56 patients with mild cognitive impairment (MCI) and $\mathrm{AD}$ when compared to 42 healthy controls using quantitative PCR (Solerte et al., 2005; Liu et al., 2014; Zhu et al., 2015). Decreased miR-210 and VEGF levels were correlated with the degree of dementia and disease severity (Zhu et al., 2015).

One of the largest studies investigating Parkinson's disease (PD) and AD miRNA biomarkers was conducted in 2014 by Burgos et al. CSF miRNA profiles and post-mortem biopsies were studied in 69 patients with $\mathrm{AD}, 67 \mathrm{PD}$ patients, and 78 healthy controls using next generation sequencing. Forty-one CSF 
miRNAs were downregulated in $\mathrm{AD}$ patients when compared to controls. Seventy-three percent of the dysregulated miRNAs have been previously linked to $\mathrm{AD}$, showing reproducibility amongst miRNA studies. When compared to PD patients, only miR-32-5p was found to be unique to $\mathrm{AD}$. Seventeen miRNAs were found to be dysregulated and 14 downregulated in the CSF of PD patients compared to controls. Additionally, decreased CSF expression of miR-9-3p and miR-708-3p correlated with an increased Braak score. MiR-101-3p was also linked to amyloid precursor protein (APP) expression and Tau phosphorylation. Furthermore, Lewy body pathology progression was found to be linked to higher miR-34a-5p and miR-374a-5p CSF expression (Burgos et al., 2014).

In addition to $\mathrm{AD}, \mathrm{CSF}$ miRNA has also been studied in amyotrophic lateral sclerosis (ALS). De Felice et al. examined CSF miRNA from 10 sporadic ALS (sALS) patients and 10 controls using qRT-PCR. Several CSF miRNA were differentially expressed in sALS patients relative to controls, including miR338-3p (De Felice et al., 2014). MiR-388-3p is of particular interest because it had been previously shown to be upregulated in frontal cortex tissues, blood, leukocytes, serum, and spinal cord tissues (Shioya et al., 2010; De Felice et al., 2014; Cloutier et al., 2015). In addition, MiR-338-3p has been speculated to be involved in many neuronal functions, including neuroblast and mature neuron apoptosis as well as neuronal metabolic pathway regulation (Aschrafi et al., 2008; Ragusa et al., 2010). Elevation of other miRNAs, such as CSF miR-27b, miR-146a, and miR532-3p, cannot only be used to recognize ALS but also enable the differentiation of ALS from multiple sclerosis (Cloutier et al., 2015).

Freischmidt et al. also found a strong link between dysregulated miRNA and TDP-43 in ALS patients. Freischmidt analyzed the CSF miRNA from 22 sALS patients and 24 control subjects and found that 5 out of 9 miRNA associated with TDP43 were dysregulated in the CSF of ALS patients. MiR-132-5p, miR-132-3p, miR-143-3p were upregulated while miR-143-5p and miR-574-5p were downregulated (Freischmidt et al., 2013). TDP-43 is a central player in ALS pathogenesis and therefore its linkage with miRNAs makes miRNA even more credible as a biomarker.

Lastly, CSF miRNA profile has been examined in multiple sclerosis (MS). CSF miRNA expression was studied in 53 MS patients and 39 controls. MiR-181c and miR-633 were upregulated and miR-922 was downregulated in MS patients when compared to controls. When combined, miR-633 and miR-922 was $88 \%$ sensitive and $69 \%$ specific in MS diagnosis. Additionally, miR-181c and miR-633 could be used to differentiate relapsing-remitting (RRMS) and secondary progressive MS (SPMS) courses with a specificity of $82 \%$ specificity and sensitivity of $69 \%$. These miRNA levels were increased in RRMS when compared to SPMS, but were expressed at higher levels in all MS cases compared to controls (Haghikia et al., 2012; See Table 1).

\section{Other Conditions}

Beyond neurodegenerative disorders, CSF miRNA profiles have been explored in CNS malignancies. In a study examining 23
TABLE 1 | Potential microRNA biomarkers identified in plasma and Cerebrospinal Fluid (CSF) in different neurodegenerative diseases.

\begin{tabular}{|c|c|c|}
\hline Disorder & Increased & Decreased \\
\hline Alzheimer's disease & $\begin{array}{l}\operatorname{miR}-29 a^{a, b}, \operatorname{miR}-210^{c, d, e}, \\
\text { miR-216a }^{c}, d, \operatorname{miR}^{-} 802^{f}\end{array}$ & $\begin{array}{l}\text { miR-9g, miR-26b } b^{a, h}, \\
\text { miR-27a }^{a, c, d}, m^{a R-125 b^{a, b}, h}\end{array}$ \\
\hline Parkinson's disease & & Overall downregulation \\
\hline $\begin{array}{l}\text { Amyotrophic lateral } \\
\text { sclerosis }\end{array}$ & $\begin{array}{l}\operatorname{miR}-338-3 p^{i} \\
143-5 p^{j}\end{array}$ & $\begin{array}{l}\text { 132-3p, 132-5p, 143-3p, } \\
\text { let-7bj,k }\end{array}$ \\
\hline Multiple sclerosis & miR-181c, miR-633 & miR-633 + miR-922 \\
\hline
\end{tabular}

a Kiko et al. (2014); b Müller et al. (2015); ‘ Cogswell et al. (2008); d Sala Frigerio et al. (2013); e Zhu et al. (2015); ${ }^{f}$ van Harten et al. (2011); ${ }^{g}$ Burgos et al. (2014); ${ }^{h}$ Galimberti et al. (2014); iDe Felice et al. (2014); 'iFreischmidt et al. (2013); ${ }^{k}$ Cloutier et al. (2015).

primary CNS lymphoma (PCNSL) patients and 30 controls and a follow-up with 16 additional PCNSL patients, Baraniskin et al. found that miR-21, miR-19b, and miR-92 were among the most highly upregulated miRNA in PCNSL (Baraniskin et al., 2011). These miRNA had a95.7-97.4\% sensitivity and $96.7 \%$ specificity for PCNSL and could also differentiate PCNSL from inflammatory CNS disorders. In addition, these miRNA were found to correlate with tumor volume on MRI, as well as disease progression, remission, and relapse in response to chemotherapy (Baraniskin et al., 2011, 2012a). Of the 3 upregulated miRNA, miR-21 was the most highly elevated, with a relative expression level of 60.0 in PCNSL and 3.8 in controls (Baraniskin et al., 2011). MiR-21 is upregulated in a variety of CNS tumors, possibly because of its role in targeting tumor suppressors Acidic Nuclear Phosphoprotein 32 Family, Member A (ANP32A) and SWI/SNF-related, Matrix-associated, Actin-dependent Regulator Chromatin group A (SMARCA4) (Akers et al., 2013).

CSF miRNA profiling has also been explored in gliomas and glioblastomas. Like PCNSL, gliomas are characterized by miR-21 over-expression, with a $85-87 \%$ sensitivity and 93$100 \%$ specificity in glioblastoma detection (Gabriely et al., 2008; Baraniskin et al., 2012a; Akers et al., 2013). Akers et al. reported a decreased miR-21 level of more than 50-fold in a surgical patient as compared to pre-surgery level, suggesting that miR-21 can potentially be used to determine treatment efficacy (Akers et al., 2013). Furthermore, low expression of MiR-10b was found in normal individuals, and high expression was noted in roughly $90 \%$ of glioma subjects. Additionally, miR-10b correlates well with erlotinib treatment, CSF cytology, and MRI results (Teplyuk et al., 2012). Finally, miR-15b, can detect glioma with a $90 \%$ sensitivity and $94.9 \%$ specificity alone or a $90 \%$ sensitivity and $100 \%$ specificity when measured along with miR-21 (Baraniskin et al., 2012b).

In addition to cancers and neurodegenerative diseases, brain injuries and infections can also potentially be detected by CSF miRNA profiling. Traumatic brain injuries (TBI), for example, are associated with elevated miR-let-7i and miR451 , which are thought to produce TBI-associated damages through the action of cytokines S100b and UCH L1. TBIs are also associated with decreased miR-9 levels and higher overall CSF miRNA levels (Balakathiresan et al., 2012; Patz et al., 2013). HIV encephalitis (HIVE) patients, while they do not exhibit any higher overall CSF miRNA, do show increased 
levels of miR-937, miR-598 and miR-595 (Pacifici et al., 2013). Moreover, stroke patients have been shown to exhibit elevated miR-let-7c and miR-221-3p (Sørensen et al., 2014). Psychiatric conditions, such as major depressive disorder (MDD), may also benefit from CSF miRNA detection Notably, miR-221$3 p$, miR-34a-5p, and miR-let-7d-3p levels were upregulated and miR-451 was downregulated with a sensitivity of $84.85-$ $96.88 \%$ and specificity of $90.48-95.24 \%$ for MDD (Wan et al., 2015).

\section{FUTURE DIRECTIONS}

MiRNA CSF profiling may lead to early diagnosis of disease, allowing for early prevention implementation and timely treatment of many disorders. For example, diffuse neuronal damage in TBI may be identified using miRNA biomarkers when CT scans are negative (Balakathiresan et al., 2012; Rao et al., 2013). MiRNA may also guide prognosis and help determine the aggressiveness of the required treatment, such as suicide risk-stratification in MDD or tumor staging (Baraniskin et al., 2012a; Wan et al., 2015). Longitudinal miRNA examination may allow for tracking of disease progression and treatment efficacy (Baraniskin et al., 2012a). These, and many other applications of miRNA CSF profiling have yet to be fully explored.

Altered miRNA expression has been noted in the serum and brain tissue samples of patients with frontotemporal dementia, progressive supranuclear palsy, bipolar affective disorder, addiction/substance abuse, anxiety disorders, autism, Tourette's syndrome, postoperative delirium, oligodendroma, astrocytoma and medulloblastoma. Specific miRNA have also been implicated in some of these disease processes, and show promise for further examination as CSF biomarkers (De Smaele et al., 2010; Chan and Kocerha, 2012; Stoicea et al., 2013; Kolshus et al., 2014; Ruggeri et al., 2014). Additionally, miRNA CSF have been studied in schizophrenia, but the small sample size precluded comparison between diseased and control CSF levels (Gallego et al., 2012).

A meta-analysis concluded that the strongest singular CSF miRNA biomarker for glioma, miR-21, achieved $82 \%$ sensitivity and $94 \%$ specificity. However, pooled results of all miRNA studied and taken as a diagnostic panel, had a sensitivity of $91 \%$ sensitivity and specificity of 94\% (Qu et al., 2015). Additionally, miRNA combinations are sometimes required to differentiate one particular CNS disease from another since miRNAs have such widespread action. While increased miR-21 and miR$10 \mathrm{~b}$ are indicative of various types of brain cancer, relatively increased miR-200 signifies metastasis over primary tumor. When 7 miRNAs (miR-10b, miR-21, miR-125b, miR-141, and several miR-200s) were studied as a panel, diagnostic probability was higher at 99\% than single biomarkers (Teplyuk et al., 2012).

Many studies searched miRNA gene banks to connect miRNAs with disease-related proteins. Matches either supported previously hypothesized disease processes or proposed new processes (Gabriely et al., 2008; Hébert et al., 2008; Robertus et al., 2009; Medina et al., 2010; Balakathiresan et al., 2012; Wan et al., 2015). Continued research on miRNA and its function may further elucidate miRNA links to disease pathology and disease-causing genetic imbalances could be restored with miRNA-targeted treatment (Baraniskin et al., 2012b; Müller et al., 2015; Wan et al., 2015; Zhu et al., 2015). Oved et al. concluded that treatment of human cell tissue lines with paroxetine decreased miR-221 and miR-222 (Oved et al., 2013). Additionally, miravirsen, an anti-miR-122 treatment, is currently in Phase 2 clinical trials for the treatment of chronic hepatitis C (Kolshus et al., 2014).

Although this review presents strong evidence for the clinical importance of CSF biomarkers, there are a few limitations. Among the various studies, there was significant control group variation in number, as CSF can be difficult to obtain in healthy individuals (Baraniskin et al., 2011, 2012b; Haghikia et al., 2012; Sørensen et al., 2014; Müller et al., 2015; Zhu et al., 2015). Also, there isn't a control group data base for miRNA expression that would normalize background variability for disease profile comparison. Additionally, most of the studies had small sample sizes, some with as few as 6 patients (Sørensen et al., 2014). Larger scale and longitudinal studies must be completed to further validate these results before clinical integration. Repetition would also help identify the most sensitive and specific miRNA biomarkers, as some studies show differing results, potentially from varying methods of analysis (Cogswell et al., 2008; Müller et al., 2015; Zhu et al., 2015). Furthermore, it should be noted that certain medications, including some anesthetics, can affect miRNA levels and potentially complicate results (Ackermann et al., 2014). Finally, lumbar puncture is not currently standard diagnostic procedure for many of these conditions, including PCNSL, glioma, TBI and depression (Baraniskin et al., 2011, 2012b; Balakathiresan et al., 2012; Wan et al., 2015).

Additionally, various methods are used in miRNA acquisition, extraction, amplification, processing, quantification, normalization and statistical analysis. This is even further complicated since miRNA's stability allows analysis from preserved, frozen, and fresh tissues and fluids (Xi et al., 2007; Weber et al., 2010). However, sample quality, time elapsed since collection, and storage methods can all affect results (De Smaele et al., 2010). The various extraction methods and reagents used yield different miRNA levels and profiles (Eldh et al., 2012). Further discrepancies may result from the multitude of data processing software and data normalization techniques available for use (Meyer et al., 2010; Garber et al., 2011).

Although new techniques are being developed, there are three major approaches to miRNA-specific quantification: quantitative reverse transcription polymerase chain reaction (qrPCR), hybridization-based methods using arrays, and highthroughput sequencing or next generation sequencing (nextgen). Each technique has its own strengths and weaknesses. QrPCR provides the most accurate absolute miRNA quantification and is already widely used in DNA and RNA processing. However, reaction conditions can differ between machines and samples, and it is not ideal for novel miRNA identification. This is the least expensive miRNA analysis method (Pritchard et al., 2012). Microarrays of fluorescent probes or beads are best used to compare miRNA levels between samples, but are less accurate, sensitive, and reliable for absolute quantification because non-identical sequences can hybridize (Pritchard et al., 
2012). This well-established method requires a larger amount of starting miRNA (De Smaele et al., 2010). Nextgen is a promising new technique that measures relative miRNA expression, as opposed to direct absolute quantification. It can accurately differentiate similar miRNAs and identify novel miRNA. Nextgen quantification is also able to yield a massive amount of data for computation. Methods other than nextgen-like techniques require previous knowledge about the miRNA in question, so results can be limited and biased. As a new technology, nextgen is the most expensive miRNA analysis method (Meyer et al., 2010)

\section{CONCLUSION}

Although a newly emerging field, CSF miRNA sampling and expression profiling show promise as a noninvasive CNS biomarker. MiRNAs exhibit widespread genetic action, playing a role in many cellular functions, and have been linked to pathological processes. MiRNA changes are often detectable prior to their downstream protein expression effects. They are protected and stable in various body fluids and tissues, making them a valuable biomarker. While serum miRNA can be a useful biomarker in various other diseases, the BBB might preclude its use in the diagnosis of neurological disorders. CSF, a promising alternative, is a relatively cell- and microorganism-free fluid.

\section{REFERENCES}

Ackermann, W., Stoicea, N., Wojda, T. R., Steiner, N., Barnett, C. J., and Bergese, S. D. (2014). Does post-operative cognitive decline promote/accelerate Alzheimer's disease pathology? Perioperative cerebrospinal fluid biomarkers and the gap of knowledge. Aust. J. Anesth. Anal. 2:1038.

Akers, J. C., Ramakrishnan, V., Kim, R., Skog, J., Nakano, I., Pingle, S., et al. (2013). MiR-21 in the extracellular vesicles (EVs) of cerebrospinal fluid (CSF): a platform for glioblastoma biomarker development. PLOS ONE 8:e78115. doi: 10.1371/journal.pone.0078115

Alexandrov, P. N., Dua, P., Hill, J. M., Bhattacharjee, S., Zhao, Y., and Lukiw, W. J. (2012). microRNA (miRNA) speciation in Alzheimer's disease (AD) cerebrospinal fluid (CSF) and extracellular fluid (ECF). Int. J. Biochem. Mol. Biol. 3, 365-373.

Aschrafi, A., Schwechter, A. D., Mameza, M. G., Natera-Naranjo, O., Gioio, A. E., and Kaplan, B. B. (2008). MicroRNA-338 regulates local cytochrome c oxidase IV mRNA levels and oxidative phosphorylation in the axons of sympathetic neurons. J. Neurosci. 28, 12581-12590. doi: 10.1523/JNEUROSCI. 3338-08.2008

Balakathiresan, N., Bhomia, M., Chandran, R., Chavko, M., McCarron, R. M., and Maheshwari, R. K. (2012). MicroRNA let-7i is a promising serum biomarker for blast-induced traumatic brain injury. J. Neurotrauma 29, 1379-1387. doi: 10.1089/neu.2011.2146

Baraniskin, A., Kuhnhenn, J., Schlegel, U., Chan, A., Deckert, M., Gold, R., et al. (2011). Identification of microRNAs in the cerebrospinal fluid as marker for primary diffuse large B-cell lymphoma of the central nervous system. Blood 117, 3140-3146. doi: 10.1182/blood-2010-09-308684

Baraniskin, A., Kuhnhenn, J., Schlegel, U., Maghnouj, A., Zöllner, H., Schmiegel, W., et al. (2012b). Identification of microRNAs in the cerebrospinal fluid as biomarker for the diagnosis of glioma. Neuro-oncology 14, 29-33. doi: 10.1093/neuonc/nor169

Baraniskin, A., Kuhnhenn, J., Schlegel, U., Schmiegel, W., Hahn, S., and Schroers, R. (2012a). MicroRNAs in cerebrospinal fluid as biomarker for disease course monitoring in primary central nervous system lymphoma. J. Neurooncol. 109, 239-244. doi: 10.1007/s11060-012-0908-2
The CNS is the least surgically accessible body system, making diagnosis of disorders invasive, costly, and potentially dangerous. Lumbar puncture is already part of the diagnostic workup of many neurodegenerative diseases including $\mathrm{AD}$ and PD. That being said, miRNA CSF profiling would be relatively easy to integrate into the management of these diseases. However, the current diagnostic workup of psychiatric disorders and brain cancers relies primarily on clinical examination and invasive brain biopsy. While currently, miRNA CSF profiling focuses mainly on disease identification, there are numerous other applications including prognosis, course tracking, and potential treatment of CNS diseases. Alongside patient history, physical exam, and clinical testing, miRNA profiles have the potential to contribute positively to patient care and outcomes.

\section{AUTHOR CONTRIBUTIONS}

The authors declare equal contribution in the writing, editing and final version of the present manuscript.

\section{ACKNOWLEDGMENTS}

Nicholas Joseph from The Ohio State University Department of Neuroscience contributed during the editing process.

Begley, D. J., and Brightman, M. W. (2003). Structural and functional aspects of the blood-brain barrier. Prog. Drug Res. 6, 19-78. doi: 10.1007/978-3-034 8-8049-7_2

Berzin, T. M., Zipser, B. D., Rafii, M. S., Kuo-Leblanc, V., Yancopoulos, G. D., Glass, D. J., et al. (2000). Agrin and microvascular damage in Alzheimer's disease. Neurobiol. Aging 21, 349-355. doi: 10.1016/S0197-4580(00) 00121-4

Blennow, K., Wallin, A., Fredman, P., Karlsson, I., Gottfries, C. G., and Svennerholm, L. (1990). Blood-brain barrier disturbance in patients with Alzheimer's disease is related to vascular factors. Acta Neurol. Scand. 81, 323-326. doi: 10.1111/j.1600-0404.1990.tb01563.x

Bonauer, A., Boon, R. A., and Dimmeler, S. (2010). Vascular micrornas. Curr. Drug Targets 11, 943-949. doi: 10.2174/138945010791591313

Brinkmalm, A., Brinkmalm, G., Honer, W. G., Frölich, L., Hausner, L., Minthon, L., et al. (2014). SNAP-25 is a promising novel cerebrospinal fluid biomarker for synapse degeneration in Alzheimer's disease. Mol. Neurodegener. 9:53. doi: 10.1186/1750-1326-9-53

Burgos, K. L., Javaherian, A., Bomprezzi, R., Ghaffari, L., Rhodes, S., Courtright, A., et al. (2013). Identification of extracellular miRNA in human cerebrospinal fluid by next-generation sequencing. RNA 19, 712-722. doi: 10.1261/rna.036863.112

Burgos, K., Malenica, I., Metpally, R., Courtright, A., Rakela, B., Beach, T., et al. (2014). Profiles of extracellular miRNA in cerebrospinal fluid and serum from patients with Alzheimer's and Parkinson's diseases correlate with disease status and features of pathology. PLoS ONE 9:e94839. doi: 10.1371/journal.pone.0094839

Calin, G. A., Dumitru, C. D., Shimizu, M., Bichi, R., Zupo, S., Noch, E., et al. (2002). Frequent deletions and down-regulation of micro- RNA genes miR15 and miR16 at 13q14 in chronic lymphocytic leukemia. Proc. Natl. Acad. Sci. U.S.A. 99, 15524-15529. doi: 10.1073/pnas.242606799

Chan, A. W., and Kocerha, J. (2012). The path to microRNA therapeutics in psychiatric and neurodegenerative disorders. Front. Genet. 3:82. doi: 10.3389/fgene.2012.00082

Cheng, L., Quek, C. Y., Sun, X., Bellingham, S. A., and Hill, A. F. (2013). The detection of microRNA associated with Alzheimer's disease in biological 
fluids using next-generation sequencing technologies. Front. Genet. 4:150. doi: $10.3389 /$ fgene. 2013.00150

Cloutier, F., Marrero, A., O'Connell, C., and Morin, P., Jr. (2015). MicroRNAs as potential circulating biomarkers for amyotrophic lateral sclerosis. J. Mol. Neurosci. 56,102-112. doi: 10.1007/s12031-014-0471-8

Cogswell, J. P., Ward, J., Taylor, I. A., Waters, M., Shi, Y., Cannon, B., et al. (2008). Identification of miRNA changes in Alzheimer's disease brain and CSF yields putative biomarkers and insights into disease pathways. J. Alzheimers Dis. 14, 27-41.

Czeiter, E., Mondello, S., Kovacs, N., Sandor, J., Gabrielli, A., Schmid, K., et al. (2012). Brain injury biomarkers may improve the predictive power of the IMPACT outcome calculator. J. Neurotrauma 29, 1770-1778. doi: 10.1089/neu.2011.2127

De Felice, B., Annunziata, A., Fiorentino, G., Borra, M., Biffali, E., Coppola, C., et al. (2014). miR-338-3p is over-expressed in blood, CFS, serum and spinal cord from sporadic amyotrophic lateral sclerosis patients. Neurogenetics 15, 243-253. doi: 10.1007/s10048-014-0420-2

De Smaele, E., Ferretti, E., and Gulino, A. (2010). MicroRNAs as biomarkers for CNS cancer and other disorders. Brain Res. 133, 800-811. doi: 10.1016/j.brainres.2010.03.103

Eldh, M., Lötvall, J., Malmhäll, C., and Ekström, K. (2012). Importance of RNA isolation methods for analysis of exosomal RNA: evaluation of different methods. Mol. Immunol. 50, 278-286. doi: 10.1016/j.molimm.2012.02.001

Ferretti, E., De Smaele, E., Miele, E., Laneve, P., Po, A., Pelloni, M., et al. (2008). Concerted microRNA control of Hedgehog signalling in cerebellar neuronal progenitor and tumour cells. EMBO J. 27, 2616-2627. doi: 10.1038/emboj.2008.172

Freischmidt, A., Müller, K., Ludolph, A. C., and Weishaupt, J. H. (2013). Systemic dysregulation of TDP-43 binding microRNAs in amyotrophic lateral sclerosis. Acta Neuropathol. Commun. 1:42. doi: 10.1186/2051-5960-1-42

Gabriely, G., Wurdinger, T., Kesari, S., Esau, C. C., Burchard, J., Linsley, P. S., et al. (2008). MicroRNA 21 promotes glioma invasion by targeting matrix metalloproteinase regulators. Mol. Cell. Biol. 28, 5369-5380. doi: 10.1128/MCB.00479-08

Galimberti, D., Villa, C., Fenoglio, C., Serpente, M., Ghezzi, L., Cioffi, S. M., et al. (2014). Circulating miRNAs as potential biomarkers in Alzheimer's disease. J. Alzheimers. Dis. 42, 1261-1267. doi: 10.1016/j.jalz.2014.05.278

Gallego, J. A., Gordon, M. L., Claycomb, K., Bhatt, M., Lencz, T., and Malhotra, A. $\mathrm{K}$. (2012). In vivo microRNA detection and quantitation in cerebrospinal fluid. J. Mol. Neurosci. 47, 243-248. doi: 10.1007/s12031-012-9731-7

Garber, M., Grabherr, M. G., Guttman, M., and Trapnell, C. (2011). Computational methods for transcriptome annotation and quantification using RNA-seq. Nat. Methods 8, 469-477. doi: 10.1038/nmeth.1613

Ghidoni, R., Benussi, L., Paterlini, A., Albertini, V., Binetti, G., and Emanuele, E. (2011). Cerebrospinal fluid biomarkers for Alzheimer's disease: the present and the future. Neurodegener. Dis. 8, 413-420. doi: 10.1159/000327756

Guan, Y., Mizoguchi, M., Yoshimoto, K., Hata, N., Shono, T., Suzuki, S. O., et al. (2010). MiRNA-196 is upregulated in glioblastoma but not in anaplastic astrocytoma and has prognostic significance. Clin. Cancer Res. 16, 4289-4297. doi: 10.1158/1078-0432.CCR-10-0207

Haghikia, A., Haghikia, A., Hellwig, K., Baraniskin, A., Holzmann, A., Decard, B. F., et al. (2012). Regulated microRNAs in the CSF of patients with multiple sclerosis: a case-control study. Neurology 79, 2166-2170. doi: 10.1212/WNL.0b013e3182759621

Hébert, S. S., Horré, K., Nicolaï, L., Papadopoulou, A. S., Mandemakers, W., Silahtaroglu, A. N., et al. (2008). Loss of microRNA cluster miR-29a/b-1 in sporadic Alzheimer's disease correlates with increased BACE1/betasecretase expression. Proc. Natl. Acad. Sci. U.S.A. 105, 6415-6420. doi: 10.1073/pnas.0710263105

Hoglund, K., Fourier, A., Perret-Liaudet, A., Zetterberg, H., Blennow, K., and Portelius, E. (2015). Alzheimer's disease - Recent biomarker developments in relation to updated diagnostic criteria. Clin. Chim. Acta 44, 9-8. doi: 10.1016/j.cca.2015.01.041

Humpel, C. (2011). Identifying and validating biomarkers for Alzheimer's disease. Trends Biotechnol. 29, 26-32. doi: 10.1016/j.tibtech.2010.09.007

Kern, S., Skoog, I., Borjesson-Hanson, A., Blennow, K., Zetterberg, H., Ostling, S., et al. (2014). Higher CSF interleukin-6 and CSF interleukin-8 in current depression in older women. Results from a population-based sample. Brain Behav. Immun. 4, 15-18. doi: 10.1016/j.bbi.2014.05.006

Kiko, T., Nakagawa, K., Tsuduki, T., Furukawa, K., Arai, H., and Miyazawa, T. (2014). MicroRNAs in plasma and cerebrospinal fluid as potential markers for Alzheimer's disease. J. Alzheimers. Dis. 39, 253-259. doi: 10.3233/JAD-130932

Kim, D. H., Yeo, S. H., Park, J. M., Choi, J. Y., Lee, T. H., Park, S. Y., et al. (2014). Genetic markers for diagnosis and pathogenesis of Alzheimer's disease. Gene 545, 185-193. doi: 10.1016/j.gene.2014.05.031

Kolshus, E., Dalton, V. S., Ryan, K. M., and McLoughlin, D. M. (2014). When less is more-microRNAs and psychiatric disorders. Acta Psychiatr. Scand. 129, 241-256. doi: 10.1111/acps.12191

Ksiazek-Winiarek, D. J., Kacperska, M. J., and Glabinski, A. (2013). MicroRNAs as novel regulators of neuroinflammation. Mediators Inflamm. 201, 372351. doi: $10.1155 / 2013 / 172351$

Kvartsberg, H., Duits, F. H., Ingelsson, M., Andreasen, N., Öhrfelt, A., Andersson, K., et al. (2014). Cerebrospinal fluid levels of the synaptic protein neurogranin correlates with cognitive decline in prodromal Alzheimer's disease. Alzheimers Dement. 11, 1180-1190. doi: 10.1016/j.jalz.2014.10.009

Lehmann, S. M., Krüger, C., Park, B., Derkow, K., Rosenberger, K., Baumgart, J., et al. (2012). An unconventional role for miRNA: let-7 activates Toll-like receptor 7 and causes neurodegeneration. Nat. Neurosci. 15, 827-835. doi: 10.1038/nn.3113

Liu, P., Li, Y. W., Wang, X. S., Zou, X., Zhang, D. Z., Wang, D. X., et al. (2013). High serum interleukin-6 level is associated with increased risk of delirium in elderly patients after noncardiac surgery: a prospective cohort study. Chin. Med. J. 126, 3621-3627.

Liu, S. C., Chuang, S. M., Hsu, C. J., Tsai, C. H., Wang, S. W., and Tang, C. H. (2014). CTGF increases vascular endothelial growth factor-dependent angiogenesis in human synovial fibroblasts by increasing miR-210 expression. Cell Death Dis. 5:e1485. doi: 10.1038/cddis.2014.453

Maxeiner, H. G., Marion Schneider, E., Kurfiss, S. T., Brettschneider, J., Tumani, H., and Bechter, K. (2014). Cerebrospinal fluid and serum cytokine profiling to detect immune control of infectious and inflammatory neurological and psychiatric diseases. Cytokine 69, 62-67. doi: 10.1016/j.cyto.2014. 05.008

Medina, P. P., Nolde, M., and Slack, F. J. (2010). OncomiR addiction in an in vivo model of microRNA-21-induced pre-B-cell lymphoma. Nature 467, 86-90. doi: 10.1038 /nature09284

Meyer, S. U., Pfaffl, M. W., and Ulbrich, S. E. (2010). Normalization strategies for microRNA profiling experiments: a 'normal' way to a hidden layer of complexity? Biotechnol. Lett. 32, 1777-1788. doi: 10.1007/s10529-010-0380-Z

Müller, M., Jäkel, L., Bruinsma, I. B., Claassen, J. A., Kuiperij, H. B., and Verbeek, M. M. (2015). MicroRNA-29a is a candidate biomarker for Alzheimer's disease in cell-free cerebrospinal fluid. Mol. Neurobiol. doi: 10.1007/s12035-015-91568. [Epub ahead of print].

Oved, K., Morag, A., Pasmanik-Chor, M., Rehavi, M., Shomron, N., and Gurwitz, D. (2013). Genome-wide expression profiling of human lymphoblastoid cell lines implicates integrin beta-3 in the mode of action of antidepressants. Transl. Psychiatry 3:e313. doi: 10.1038/tp.2013.86

Pacifici, M., Delbue, S., Ferrante, P., Jeansonne, D., Kadri, F., Nelson, S., et al. (2013). Cerebrospinal fluid miRNA profile in HIV-encephalitis. J. Cell. Physiol. 228, 1070-1075. doi: 10.1002/jcp.24254

Patz, S., Trattnig, C., Grunbacher, G., Ebner, B., Gülly, C., Novak, A., et al. (2013). More than cell dust: microparticles isolated from cerebrospinal fluid of brain injured patients are messengers carrying mRNAs, miRNAs, and proteins. J. Neurotrauma 30, 1232-1242. doi: 10.1089/neu. 2012.2596

Polman, C. H., Reingold, S. C., Edan, G., Filippi, M., Hartung, H. P., Kappos, L., et al. (2005). Diagnostic criteria for multiple sclerosis: 2005 revisions to the “McDonald Criteria." Ann. Neurol. 58, 840-846. doi: 10.1002/ana.20703

Pritchard, C. C., Cheng, H. H., and Tewari, M. (2012). MicroRNA profiling: approaches and considerations. Nat. Rev. Genet. 13, 358-369. doi: $10.1038 / \operatorname{nrg} 3198$

Pyykö, O., Lumela, M., Rummukainen, J., Nerg, O., Seppälä, T. T., Herukka, S., et al. (2014). Cerebrospinal fluid biomarker and brain biopsy findings in idiopathic normal pressure hydrocephalus. PLoS ONE 9:e91974. doi: 10.1371/journal.pone.0091974 
Qu, S., Guan, J., and Liu, Y. (2015). Identification of microRNAs as novel biomarkers for glioma detection: a meta-analysis based on 11 articles. J. Neurol. Sci. 348, 181-187. doi: 10.1016/j.jns.2014.11.036

Ragusa, M., Majorana, A., Banelli, B., Barbagallo, D., Statello, L., Casciano, I., et al. (2010). MIR152, MIR200B, and MIR338, human positional and functional neuroblastoma candidates, are involved in neuroblast differentiation and apoptosis. J. Mol. Med. 88, 1041-1053. doi: 10.1007/s00109-0100643-0

Rao, P., Benito, E., and Fischer, A. (2013). MicroRNAs as biomarkers for CNS disease. Front. Mol. Neurosci. 6:39. doi: 10.3389/fnmol.2013.00039

Robertus, J. L., Harms, G., Blokzijl, T., Booman, M., de Jong, D., van Imhoff, G., et al. (2009). Specific expression of miR-17-5p and miR-127 in testicular and central nervous system diffuse large B-cell lymphoma. Mod. Pathol. 22, 547-555. doi: 10.1038/modpathol.2009.10

Rosén, C., Farahmand, B., Skillbäck, T., Nägga, K., Mattsson, N., Kilander, L., et al. (2015). Benchmarking biomarker-based criteria for Alzheimer's disease: data from the Swedish Dementia Registry, SveDem. Alzheimers Dement. 11, 1470-1479. doi: 10.1016/j.jalz.2015.04.007

Ruggeri, B., Sarkans, U., Schumann, G., and Persico, A. M. (2014). Biomarkers in autism spectrum disorder: the old and the new. Psychopharmacology 231, 1201-1216. doi: 10.1007/s00213-013-3290-7

Sala Frigerio, C., Lau, P., Salta, E., Tournoy, J., Bossers, K., Vandenberghe, R., et al. (2013). Reduced expression of hsa-miR-27a-3p in CSF of patients with Alzheimer disease. Neurology 81, 2103-2106. doi: 10.1212/01.wnl.0000437306.37850.22

Schramedei, K., Mörbt, N., Pfeifer, G., Läuter, J., Rosolowski, M., Tomm, J. M., et al. (2011). MicroRNA-21 targets tumor suppressor genes ANP32A and SMARCA4. Oncogene 30, 2975-2985. doi: 10.1038/onc.2011.15

Scollato, A., Terreni, A., Caldini, A., Salvadori, B., Gallina, P., Francese, S., et al. (2010). CSF proteomic analysis in patients with normal pressure hydrocephalus selected for the shunt: CSF biomarkers of response to surgical treatment. Neurol. Sci. 31, 283-291. doi: 10.1007/s10072-009-0181-0

Sheinerman, K. S., and Umansky, S. R. (2013). Circulating cell-free microRNA as biomarkers for screening, diagnosis and monitoring of neurodegenerative diseases and other neurologic pathologies. Front. Cell. Neurosci. 7:150. doi: $10.3389 /$ fncel.2013.00150

Shioya, M., Obayashi, S., Tabunoki, H., Arima, K., Saito, Y., Ishida, T., et al. (2010). Aberrant microRNA expression in the brains of neurodegenerative diseases: miR-29a decreased in Alzheimer disease brains targets neurone navigator 3. Neuropathol. Appl. Neurobiol. 36, 320-330. doi: 10.1111/j.13652990.2010.01076.x

Siegel, S. R., Mackenzie, J., Chaplin, G., Jablonski, N. G., and Griffiths, L. (2012). Circulating microRNAs involved in multiple sclerosis. Mol. Biol. Rep. 39, 6219-6225. doi: 10.1007/s11033-011-1441-7

Solerte, S. B., Ferrari, E., Cuzzoni, G., Locatelli, E., Giustina, A., Zamboni, M., et al. (2005). Decreased release of the angiogenic peptide vascular endothelial growth factor in Alzheimer's disease: recovering effect with insulin and DHEA sulfate. Dement. Geriatr. Cogn. Disord. 19, 1-10. doi: 10.1159/0000 80963

Sørensen, S., Nygaard, A.-B., Nielsen, M.-Y., Jensen, K., and Christensen, T. (2014). miRNA expression profiles in cerebrospinal fluid and blood of patients with acute ischemic stroke. Transl. Stroke Res. 5, 711-718. doi: 10.1007/s12975014-0364-8

Stoicea, N., Wilson, T., Chandrasekhar, K., and Bergese, S. D. (2013). The role of microRNAs as potential biomarkers in central nervous system disorders. Int. J. Clin. Anesthesiol. 1:1009. doi: 10.3389/fnmol.2013.00039
Teplyuk, N. M., Mollenhauer, B., Gabriely, G., Giese, A., Kim, E., Smolsky, M., et al. (2012). MicroRNAs in cerebrospinal fluid identify glioblastoma and metastatic brain cancers and reflect disease activity. Neuro-oncology 14, 689-700. doi: $10.1093 /$ neuonc/nos074

The ALS Association. (2015). Symptoms and Diagnosis: The ALS Association; 2015 [cited 2015 October 16, 2015]. Available online at: http://www.alsa.org/aboutals/symptoms.html

van den Boogaard, M., Kox, M., Quinn, K. L., van Achterberg, T., van der Hoeven, J. G., Schoonhoven, L., et al. (2011). Biomarkers associated with delirium in critically ill patients and their relation with long-term subjective cognitive dysfunction; indications for different pathways governing delirium in inflamed and noninflamed patients. Crit Care 15, R297. doi: 10.1186/ cc10598

van Harten, A., Mulders, J., Çevik, C., Kester, M., Scheltens, P., van der Flier, W., et al. (2011). "MicroRNA analysis in the spinal fluid of Alzheimer patients: a methodological feasibility study," in Circulating Nucleic Acids in Plasma and Serum, ed P. B. Gahan (Dordrecht: Springer), 275-282.

van Munster, B. C., Korevaar, J. C., Zwinderman, A. H., Levi, M., Wiersinga, W. J., and De Rooij, S. E. (2008). Time-course of cytokines during delirium in elderly patients with hip fractures. J. Am. Geriatr. Soc. 56, 1704-1709. doi: 10.1111/j.1532-5415.2008.01851.x

Wan, Y., Liu, Y., Wang, X., Wu, J., Liu, K., Zhou, J., et al. (2015). Identification of differential MicroRNAs in cerebrospinal fluid and serum of patients with major depressive disorder. PLoS ONE 10:e0121975. doi: 10.1371/journal.pone.0121975

Weber, J. A., Baxter, D. H., Zhang, S., Huang, D. Y., Huang, K. H., Lee, M. J., et al. (2010). The microRNA spectrum in 12 body fluids. Clin. Chem. 56, 1733-1741. doi: 10.1373/clinchem.2010.147405

Wei, D., Wan, Q., Li, L., Jin, H., Liu, Y., Wang, Y., et al. (2015). MicroRNAs as potential biomarkers for diagnosing cancers of central nervous system: a meta-analysis. Mol. Neurobiol. 51, 1452-1461. doi: 10.1007/s12035-014-8822-6

Xi, Y., Nakajima, G., Gavin, E., Morris, C. G., Kudo, K., Hayashi, K., et al. (2007). Systematic analysis of microRNA expression of RNA extracted from fresh frozen and formalin-fixed paraffin-embedded samples. RNA 13, 1668-1674. doi: 10.1261/rna.642907

Zhu, Y., Li, C., Sun, A., Wang, Y., and Zhou, S. (2015). Quantification of microRNA-210 in the cerebrospinal fluid and serum: implications for Alzheimer's disease. Exp. Ther. Med. 9, 1013-1017. doi: 10.3892/etm. 2015.2179

Zipser, B. D., Johanson, C. E., Gonzalez, L., Berzin, T. M., Tavares, R., Hulette, C. M., et al. (2007). Microvascular injury and blood-brain barrier leakage in Alzheimer's disease. Neurobiol. Aging. 28, 977-986. doi: 10.1016/j.neurobiolaging.2006.05.016

Conflict of Interest Statement: The authors declare that the research was conducted in the absence of any commercial or financial relationships that could be construed as a potential conflict of interest.

Copyright (c) 2016 Stoicea, Du, Lakis, Tipton, Arias-Morales and Bergese. This is an open-access article distributed under the terms of the Creative Commons Attribution License (CC BY). The use, distribution or reproduction in other forums is permitted, provided the original author(s) or licensor are credited and that the original publication in this journal is cited, in accordance with accepted academic practice. No use, distribution or reproduction is permitted which does not comply with these terms. 